\title{
Review: citalopram is effective and safe for depression
}

Keller MB. Citalopram therapy for depression: a review of 10 years of European experience and data from US clinical trials.J Clin Psychiatry 2000 Dec;61:896-908.

\section{QUESTION: Is citalopram effective and safe in patients with depression?}

\section{Data sources}

Studies were identified by searching Medline (19662000), scanning bibliographies, and searching abstracts from major medical conferences. Meta-analyses were also included, and pharmacokinetics studies and case reports were used to supplement safety and tolerability data.

\section{Study selection}

English language studies were selected if they were randomised, double blind, controlled trials (RCTs) of citalopram in depressed patients.

\section{Data extraction}

Data were extracted on patient characteristics, drug dose, assessment method, and outcomes.

\section{Main results}

30 RCTs that compared citalopram with placebo, tricyclic or tetracyclic antidepressants, and selective serotonin reuptake inhibitors (SSRIs) met the selection criteria. Mean patient age was approximately 40 years. Primary efficacy measures were the Hamilton Rating Scale for Depression, the Montgomery Asberg Depression Rating Scale, or both. Placebo: 11 placebo controlled RCTs and 3 meta-analyses showed that citalopram reduced depression. Tricyclic and tetracyclic antidepressants: 10 double blind RCTs and a meta-analysis of 5 published and unpublished RCTS (294 patients) showed that citalopram and tricyclic antidepressants (TCAs) led to similar response rates. In general, citalopram had a better safety profile than the TCAs. SSRIs: Citalopram was shown to have similar efficacy to fluoxetine at 8 weeks (2 RCTs, 296 and 314 patients), sertraline at 24 weeks (1 RCT, 308 patients), and fluvoxamine at 6 weeks (1 RCT, 217 patients). 1 placebo controlled RCT (323 patients) showed that improvement occurred sooner with citalopram than with sertraline. Prevention of relapse and recurrence: 4 placebo controlled RCTs (3 in adults, 1 in elderly people) showed that citalopram is effective for preventing depression relapse and recurrence.

In the short term, citalopram led to more somnolence, nausea, and dry mouth than placebo but did not result in clinically significant central nervous system stimulant side effects. 1 long term RCT (6 mo) showed that citalopram did not lead to more side effects than placebo; another RCT of citalopram for panic disorder showed that increased sweating was the only side effect that occurred more frequently with citalopram than with placebo.

\section{Conclusions}

In patients with depression, citalopram is more effective than placebo and is as effective as tricyclic or tetracyclic antidepressants and selective serotonin reuptake inhibitors. Side effects occur less frequently with citalo- pram than with tricyclic depressants. Citalopram does not lead to more long term side effects than placebo.

\section{COMMENTARY}

In the past few years, several overviews and/or systematic reviews have tried to define the first line treatment of depression..$^{1-3}$ Consistent findings across these reviews are: (1) in general, SSRIs and TCAs do not differ in efficacy, and (2) differences in dropout rates favour SSRIs only slightly. It is well known, however, that many relevant outcomes are missing in RCTs, particularly if one wants to match patient individual characteristics and a particular antidepressant

This comprehensive review by Keller focuses on citalopram, the most recent SSRI approved for the treatment of depression in the US. Using information from double blind RCTs and data from pharmacokinetic studies and case reports, the author assesses the efficacy and tolerability of citalopram when compared with placebo, TCAs, and other SSRIs.

Although using a restrictive search strategy for locating RCTs, Keller's review has the advantage of including other study designs. Outcomes that are relevant for choosing a specific antidepressant, such as long term effects of the medication, are poorly reported and investigated in RCTs but may be available in other study designs.

As expected, citalopram is better than placebo, has similar efficacy to that of the TCAs, and is, as are other SSRIs, well tolerated and safe. Keller's approach provides further relevant information: in vitro studies suggest that citalopram has minimal effects on the cytochrome P450 system, with a low potential for drug interactions. In vivo studies have confirmed these findings, and consequently, it can be said that citalopram is a good alternative for the elderly and patients with comorbid illness.

Other interesting findings, such as the more rapid onset of action than fluoxetine, still require replication in larger RCTs. Another RCT, which was not included in this review, found a potentially faster onset of efficacy of mirtazapine over citalopram. ${ }^{4}$

Lack of scientific information from RCTs must not be paralysing for clinical decisions. This review highlights the fact that depending on the specific question, reliable evidence can be found in studies other than RCTs.

Maurício Silva de Lima, MD, MSc, $\mathrm{PhD}$ Universidade Federal de Pelotas and Universidade Católica de Pelotas Pelotas, RS, Brazil

1 Hotopf M, Lewis G, Normand C. Are SSRIs a cost-effective alternative to tricyclics? Br J Psychiatry 1996;168:404-9.

2 Song F, Freemantle N, Sheldon TA, et al. Selective serotonin reuptake inhibitors: meta-analysis of efficacy and acceptability. $B M J$ 1993;306:683-7.

3 Mulrow CD, Williams JW Jr, Trivedi M, et al. Treatment of depression-newer pharmacotherapies. Rockville, MD: Agency for Health Care Policy and Research, February 1999. AHCPR Publication No 99-E014.

4 Leinonen E, Skarstein J, Behnke K, et al, and the Nordic Antidepressant Study Group. Efficacy and tolerability of mirtazapine versus citalopram: a double-blind, randomized study in patients with majordepressive disorder. Int ClinPsychopharmacol 1999;14:329-37. 